\title{
Association of carotid atherosclerosis and recurrent cerebral infarction in the Chinese population: a meta-analysis
}

This article was published in the following Dove Press journal:

Neuropsychiatric Disease and Treatment

20 February 2017

Number of times this article has been viewed

\author{
Jianping Liu \\ Yun Zhu \\ Yuhuai Wu \\ Yan Liu \\ Zhaowei Teng \\ Yinglu $\mathrm{HaO}$
}

Department of Science and Education, Nephrology, Orthopedic and Cardiology, The People's Hospital of Yuxi City, The 6th Affiliated Hospital of Kunming Medical University, Yuxi, Yunan, People's Republic of China
Correspondence: Yinglu Hao The People's Hospital of Yuxi City, The 6th Affiliated Hospital of Kunming Medical University, Yuxi 653100, Yunan, People's Republic of China Tel +86 I38 8773 I2I2

Email yingluhaoy@163.com
Abstract: Stroke, when poor blood flow to the brain results in cell death, is the third leading cause of disability and mortality worldwide, and appears as an unequal distribution in the global population. The cumulative risk of recurrence varies greatly up to 10 years after the first stroke. Carotid atherosclerosis is a major risk factor for stroke. The aim of this study was to investigate and estimate the relationship between carotid atherosclerosis and risk of stroke recurrence in the Chinese population. We performed a systematic review and meta-analysis of randomized controlled trials published from 2000 to 2013, using the following databases: PubMed, Embase, Medline, Wanfang, and the China National Knowledge Infrastructure. The odds ratios with 95\% confidence intervals were calculated to examine this strength. A total of 22 studies, including 3,912 patients, 2,506 first-ever cases, and 1,406 recurrent cases, were pooled in this meta-analysis. Our results showed that the frequency of carotid atherosclerosis is higher in recurrent cases than that in the first-ever controls (78.88\% vs $59.38 \%)$, and the statistical analysis demonstrated significant positive association between carotid atherosclerosis and recurrent cerebral infarction (odds ratio: $2.87 ; 95 \%$ confidence interval: $2.42-3.37 ; P<0.00001$ ) in a fixed-effect model. No significant heterogeneity was observed across all studies. In conclusion, our results showed that carotid atherosclerosis was associated with increased risk of recurrent stroke. However, further well-designed research with large sample sizes is still needed to identify the clear mechanism.

Keywords: cerebral infarction, carotid atherosclerosis, meta-analysis

\section{Introduction}

Stroke is defined as a clinical syndrome of presumed vascular origin, typified by rapidly developing signs of focal or global disturbance of cerebral functions lasting $>24$ hours or leading to death. ${ }^{1,2}$ It is the leading cause of adult disability and mortality in developing countries. ${ }^{3,4}$ Eighty-five percent of strokes are due to cerebral infarction, 10\% due to primary hemorrhage, and $5 \%$ due to subarachnoid hemorrhage. ${ }^{5}$ The risk of recurrent stroke is $26 \%$ within 5 years of the first stroke and $39 \%$ within 10 years, ${ }^{6}$ which imposes a heavy burden on society and the families of the patients. Thus, there is an urgent need to explore and evaluate effective and reliable biomarkers for stroke.

Atherosclerosis in the aortic arch is a risk factor for ischemic stroke. ${ }^{7}$ It is defined as a fatty deposit that hardens on the inner wall of the arteries (atheroma) and roughens its surface, which makes the arteries susceptible to blockage, either by narrowing or by formation of a blood clot. Atherosclerotic changes in the cerebrovascular system ultimately can lead to incident stroke. ${ }^{8}$ The association between atherosclerosis in the aortic arch and stroke risk was initially established in autopsy studies and subsequently confirmed by in vivo studies. ${ }^{9,10}$ Carotid atherosclerotic changes are a marker for 
systemic atherosclerosis. ${ }^{11}$ Atherosclerotic plaque at the carotid bifurcation is the underlying cause of the majority of ischemic strokes, and the degree of carotid stenosis is strongly associated with stroke risk in symptomatic patients. ${ }^{12}$ Pathologic studies comparing symptomatic and asymptomatic carotid plaques have demonstrated that specific plaque characteristics are associated with ischemic brain injury, and the mechanisms underlying plaque instability in the carotid circulation are similar to those in the coronary circulation. ${ }^{13}$ Research has shown that patients with a first ischemic stroke are at high risk of developing recurrent stroke due to carotid atherosclerotic plaque rupture.${ }^{14}$ Liu et $a{ }^{15}$ proved that carotid plaques in patients with recurrent ischemic stroke are significantly aggravated compared with those in patients who have experienced one stroke, and monitoring carotid plaques in patients with initial stroke by MRI may be helpful for secondary stroke prevention.

The incidence of stroke in rural China has increased rapidly, particularly among middle-aged adults, along with a concurrent increase in risk factor prevalence. ${ }^{16}$ A previous study ${ }^{17}$ has revealed that, in the Chinese population, the incidence of ischemic stroke is rising annually at a rate of $8.7 \%$; this highlights the need to prioritize stroke prevention. Furthermore, atherosclerosis has been shown as an important cause of stroke in Chinese patients. ${ }^{18}$ Therefore, understanding the natural history of carotid atherosclerosis is essential in the management of patients at risk for stroke. This study investigated the correlation between carotid atherosclerosis and recurrent cerebral infarction.

\section{Materials and methods}

\section{Identification and eligibility of relevant studies}

We conducted a comprehensive literature search for relevant articles published between January 2000 and September 2013, using the following electronic databases: PubMed, Embase, Medline, Wanfang, and China National Knowledge Infrastructure. The Medical Subject Heading terms "cerebral infarction", "stroke", "brain infarction", "cerebral arterial thrombosis", "recurrence", and "carotid atherosclerosis" were employed as the key terms. The equivalent Chinese terms were used in the Chinese databases. References of retrieved articles were searched with no language restrictions. The search was focused on studies that had been conducted in the Chinese population. Only full-text articles and the most recent studies were included in this meta-analysis.

\section{Criteria for article screening}

The inclusion criteria were as follows: 1) the paper should be a random control clinical trial or before-after study. 2) The study objects are confirmed cerebral infarction cases restricted to the Chinese population. 3) The diagnostic criteria must be in accord with the revision standard of the Fourth National Cerebrovascular Disease Conference, confirmed by craniocerebral CT or MRI. 4) The recurrence group should conform to the following standards: infarction recurrence with aggravation occurring $>1$ month after the initial onset, emergence of nerve function defect symptoms, and presence of new lesions confirmed by craniocerebral CT or MRI scan. 5) When the same authors reported two or more publications on possibly the same patient populations, only the most recent or complete study was included in this meta-analysis. The exclusion criteria were 1) patients with carotid stenosis or intracranial vascular stenosis. 2) Patients aged $>80$ years. 3) Patients with cardiac cerebral embolism, hemorrhagic cerebrovascular disease, or stroke-causing tumors, accompanied by severe heart, liver, and kidney disease.

\section{Quality assessment and data extraction}

Two investigators independently assessed the quality of the included studies according to the descriptions provided by the authors of the included trials. Any disagreement was subsequently resolved by discussion with a third investigator. The following information was extracted from each article: first author, year of publication, age and number of patients with carotid atherosclerosis, and total patients.

\section{Statistical analysis}

Analyses were carried out using the Review Manager 5.2 (The Cochrane Collaboration, London, UK). The odds ratio (OR) with $95 \%$ confidence interval (CI) was used to assess the relationship between carotid atherosclerosis and recurrent cerebral infarction. The significance of the pooled OR was determined by the $Z$-test, and $P<0.05$ was considered significant. The heterogeneity for the included articles was evaluated using the Cochran's $Q$-test and $I^{2}$ statistics. When a significant heterogeneity existed across the included studies $\left(P<0.01\right.$ and $\left.I^{2}>50 \%\right)$, the random effect model was used; when there was no significant heterogeneity across the included studies ( $P>0.01$ and $I^{2}<50 \%$ ), the fixed-effect model was used. To assess whether our results were substantially influenced by the presence of any individual study, we conducted a sensitivity analysis by systematically removing each study and recalculating the significance of the result. 
A funnel plot was used to examine the publication bias. All tests were two-sided.

\section{Results}

\section{Study selection and characteristics}

The initial search with the keywords and the subject terms identified 123 publications; only 22 met the inclusion criteria and were eligible for review. Figure 1 shows the study flow. All 22 articles (21 were written in Chinese ${ }^{19-39}$ and one was written in English ${ }^{15}$ ) reported case-control studies focusing on the association between carotid atherosclerosis and recurrence of cerebral infarction in the Chinese population. A total of 3,912 patients were included in this study, involving 2,506 first-ever cases and 1,406 recurrent cases. The detailed characteristics of the included studies are shown in Table 1.

\section{Quantitative data synthesis}

In this meta-analysis, we discussed mainly the relationship between carotid atherosclerosis and recurrent cerebral infarction. As shown in Figure 2, we observed statistically significant association between carotid atherosclerosis and recurrent cerebral infarction. The data showed that patients with recurrent cerebral infarction had a higher prevalence of carotid atherosclerosis than did patients with first-ever cerebral infarction $(78.88 \%$ vs $59.38 \%)$. The overall risk estimates OR for recurrent cerebral infarction with carotid atherosclerosis was 2.87 (95\% CI: 2.42-3.37; $P<0.00001$ ) in a fixed-effect model.

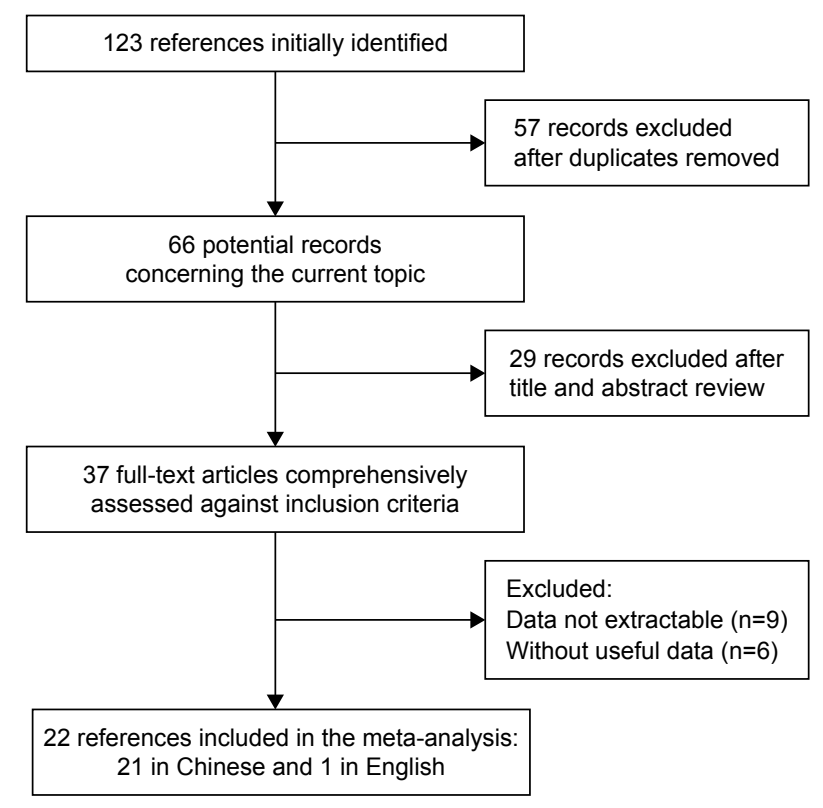

Figure I Flowchart of literature screening.

\section{Sensitivity analyses and publication bias}

A single study included in the meta-analysis was deleted each time to reflect the influence of the individual data set to the pooled ORs, and the corresponding pooled ORs were not materially changed. This procedure confirmed the stability of our overall result.

A funnel plot was constructed to assess the publication bias of the literature. The shape of the funnel plot did not reveal any evidence of funnel plot asymmetry (as shown in Figure 3).

\section{Discussion}

Stroke is one of the leading causes of death throughout the world, causing more serious chronic disabilities than any other disease. In the first several years after a stroke, the most common vascular event is another stroke. ${ }^{40}$ The risk of further stroke may be as high as 5\% within the first week and $20 \%$ within the first month. ${ }^{41}$ Therefore, timely detection and diagnosis are of clinical significance for patients with carotid atherosclerosis, particularly for preventing the occurrence and recurrence of cerebral infarction.

Carotid atherosclerosis is causally related to stroke in $>50 \%$ stenosis cases and is considered the cause of stroke in up to $20 \%$ of all cases. ${ }^{40,42}$ Studies have shown that carotid atherosclerotic plaque characteristics (seen in MRI) are related to a history of stroke and coronary heart disease $^{8}$ and correlate with predicted stroke risk. ${ }^{43}$ In the current study, we only discussed the relationship of carotid atherosclerosis excluding stenosis and recurrent cerebral infarction in the Chinese population. We found that carotid atherosclerosis accounted for $78.88 \%(1,109 / 1,406)$ of the patients with recurrent cerebral infarction. The incidence of carotid atherosclerosis in patients with recurrent cerebral infarction was higher than that with first-ever cases. The results showed that there was a statistically significant positive association between carotid atherosclerosis and recurrent cerebral infarction (OR: 2.87; 95\% CI: $2.42-3.37$, $P<0.00001)$ in a fixed-effect model.

Atherosclerosis is widely recognized as a multifactorial disease that arises from complex factors such as plaque components, blood flow, and inflammation. ${ }^{44-46}$ It has been traditionally thought that the presence of atherosclerotic plaques reduces blood flow along the carotid arteries, leading to cerebral ischemia and infarction. ${ }^{47}$ Although recent advances have identified the plaque biology, diagnosis, and treatment, atherosclerosis remains a leading cause of morbidity and mortality. ${ }^{48}$ Carotid atherosclerosis reflects 
Table I Main characteristics of included cohort studies in this meta-analysis

\begin{tabular}{|c|c|c|c|c|c|c|}
\hline \multirow{2}{*}{$\begin{array}{l}\text { References } \\
\text { (published year) }\end{array}$} & \multicolumn{3}{|l|}{ First-ever group } & \multicolumn{3}{|l|}{ Recurrence group } \\
\hline & $\begin{array}{l}\text { Age range or } \\
\text { mean } \pm \text { SD years }\end{array}$ & CA & Total & $\begin{array}{l}\text { Age range or } \\
\text { mean } \pm \text { SD years }\end{array}$ & CA & Total \\
\hline $\mathrm{Liu}^{26}(2004)$ & $64.38 \pm \mid 1.21$ & 39 & 72 & $65.25 \pm 10.32$ & 40 & 62 \\
\hline Zeng et $\mathrm{al}^{34}(2005)$ & $58.79 \pm 9.7$ & 65 & 98 & $68.53 \pm 8.25$ & 32 & 37 \\
\hline $\mathrm{Cao}^{24}(2007)$ & $58 \pm 3.0$ & 35 & 50 & $62 \pm 4.0$ & 53 & 60 \\
\hline Liu' $^{19}(2007)$ & $56 \pm 3.2$ & 55 & 80 & $60.5 \pm 4.2$ & 88 & 102 \\
\hline Yang and Chen ${ }^{21}$ (2007) & $61.4 \pm 11.0$ & 112 & 200 & $62.2 \pm 10.3$ & 230 & 266 \\
\hline Zhang $^{28}$ (2007) & $52.28 \pm 11.02$ & 30 & 53 & $56.24 \pm I 1.32$ & 41 & 55 \\
\hline Yang and $\operatorname{Lin}^{23}(2008)$ & $64.68 \pm 1.2$ & $|8|$ & 243 & $64.68 \pm 1.2$ & 19 & 25 \\
\hline Zhang and $\mathrm{Hu}^{27}$ (2008) & $46-75$ & 127 & 197 & 68.12 & 48 & 59 \\
\hline Song ${ }^{33}$ (2009) & $68 \pm 8.25$ & 99 & 203 & 66.2 & 14 & 23 \\
\hline $\operatorname{Tan}^{30}(2009)$ & $56 \pm 3.2$ & 42 & 80 & $60.5 \pm 4.2$ & 71 & 105 \\
\hline Wang $^{29}(2010)$ & $60 \pm 12$ & 27 & 63 & $72 \pm 9$ & 36 & 44 \\
\hline$W^{31}(20 I I)$ & $65.11 \pm 10.12$ & 45 & 75 & $65.37 \pm 8.09$ & 25 & 30 \\
\hline $\mathrm{Wu}$ and $\mathrm{GaO}^{32}(20 \mathrm{II})$ & - & 87 & 120 & - & 102 & 120 \\
\hline Yi et al ${ }^{39}(20 \mathrm{I} I)$ & $69.1 \pm 11.8$ & 101 & 251 & $68.1 \pm 10.9$ & 42 & 61 \\
\hline Zhao $22(201 \mathrm{I})$ & $63 \pm 9.7$ & 58 & 73 & $63 \pm 9.7$ & 42 & 45 \\
\hline Zhao et $\mathrm{al}^{25}(20 \mathrm{II})$ & - & 70 & 92 & - & 55 & 64 \\
\hline$Z_{h u}^{20}(20 I I)$ & $71.23 \pm 10.27$ & 173 & 218 & $68.91 \pm 13.15$ & 17 & 23 \\
\hline Li et $\mathrm{al}^{37}(20 \mid 2)$ & $62.78 \pm 9.62$ & 21 & 60 & $63.3 \pm 8.45$ & 38 & 48 \\
\hline Guo et $\mathrm{al}^{35}(20 \mathrm{I})$ & $65.79 \pm 9.71$ & 45 & 98 & $65.79 \pm 9.71$ & 31 & 37 \\
\hline He et a ${ }^{38}(20 \mid 2)$ & $48-79$ & 58 & 78 & $48-79$ & 46 & 52 \\
\hline Hua and $\mathrm{Zhou}^{36}(20 \mathrm{I})$ & $68.20 \pm 10.69$ & 6 & 51 & $69.84 \pm 10.08$ & 22 & 50 \\
\hline Liu et al ${ }^{15}(20 \mid 2)$ & $59.98 \pm 10.55$ & 12 & 51 & $67.05 \pm 9.69$ & 17 & 38 \\
\hline
\end{tabular}

Abbreviations: $\mathrm{CA}$, carotid atherosclerosis; SD, standard deviation.

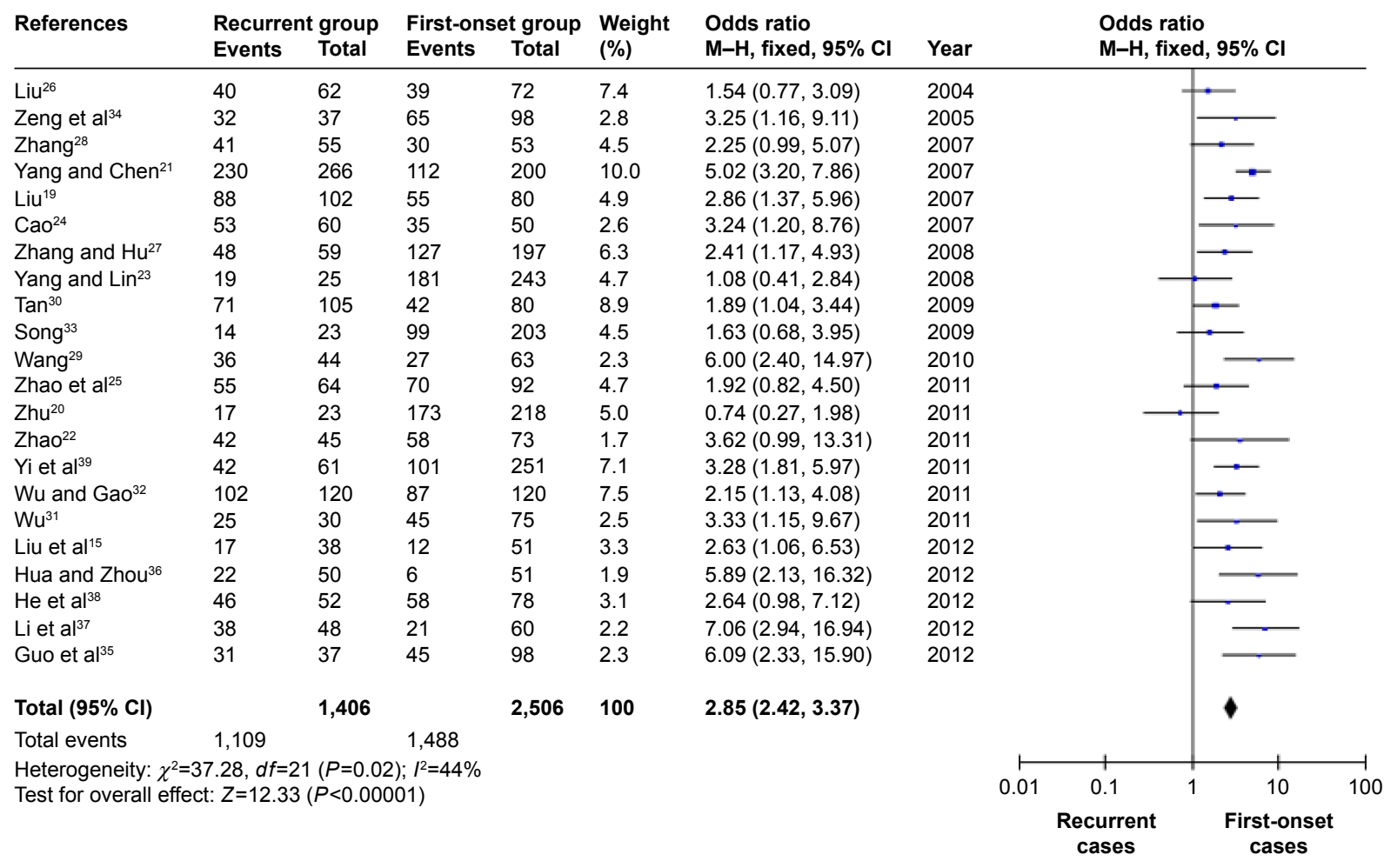

Figure 2 Forest plot of the association between carotid atherosclerosis and recurrent carotid atherosclerosis in the Chinese population. Abbreviations: $\mathrm{M}-\mathrm{H}$, Mantel-Haenszel; $\mathrm{Cl}$, confidence interval; $\mathrm{df}$, degrees of freedom. 


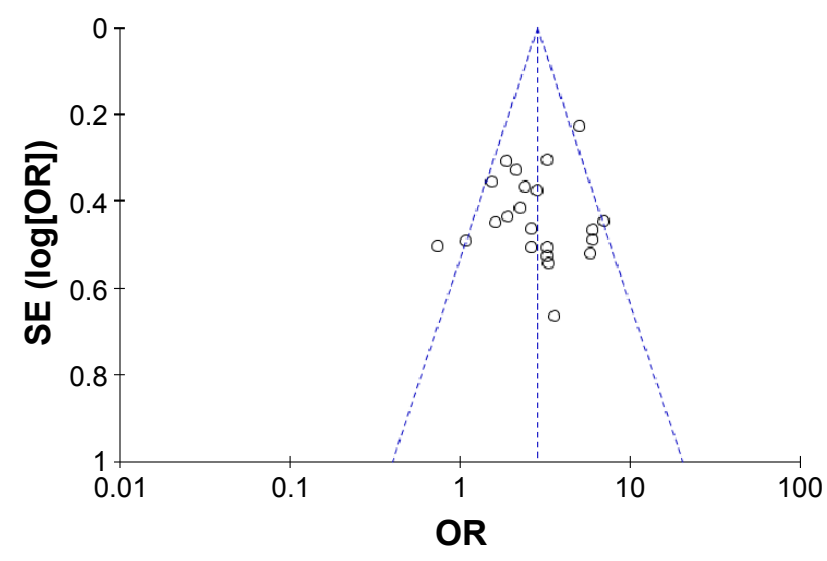

Figure 3 Funnel plot of the association between carotid atherosclerosis and recurrent carotid atherosclerosis.

Abbreviations: SE, standard error; OR, odds ratio.

generalized atherosclerosis. Carotid atherosclerosis plaque is significant in the formation of cerebral infarction. ${ }^{49,50}$ Research has proven that a slight progression of carotid atherosclerosis significantly and positively increases the prevalence of cardiovascular disease and the risk of future cardiovascular disease events..$^{51,52}$ Moreover, intima-media thickness of the carotid arteries is a marker of atherosclerosis, as has been assessed pathologically. ${ }^{53,54}$

During the last several decades, numerous studies identified the relationship between carotid atherosclerosis and recurrent cerebral infarction. ${ }^{55-57}$ Touboul et al ${ }^{55}$ found, using systematic screening, that $45 \%$ of stroke patients had carotid atherosclerosis. Nagai et $\mathrm{a}^{56}$ showed that, in addition to advanced stenosis, earlier stages of carotid atherosclerosis are associated with the increased risk for stroke. Clinical usefulness of carotid ultrasound could also improve stroke risk assessment. ${ }^{57}$ Many factors that play a role in stroke are associated with carotid atherosclerosis. Lukasik et $\mathrm{al}^{58}$ demonstrated that diminished expression of active GPIIb/IIIa in the ADP-activated platelets is associated with increased carotid intima-media thickness, independently of stroke. Markus et al ${ }^{59}$ identified that the histone deacetylase 9 genetic variant associated with ischemic stroke increases risk via promoting carotid atherosclerosis. Liu et $a^{60}{ }^{60}$ first showed that the microRNA-single nucleotide polymorphism at PON1 could affect genetic expression and is associated with an elevated risk for ischemic stroke and subclinical atherosclerosis. Furthermore, carotid atherosclerosis is associated with cognitive test scores of stroke patients. Another study ${ }^{61}$ has proved that subclinical carotid atherosclerosis, measured as the presence of plaques, number of plaques, and total plaque area, were independent long-term predictors of lower cognitive test scores. Carotid atherosclerosis markers have been associated with MRI indices of brain ischemia and aging, and with cognitive impairment in a community-based sample of middle-aged adults. ${ }^{62}$

Overall, our study demonstrated that the incidence of carotid atherosclerosis in patients with recurrent cerebral infarction was higher than that in first-ever cases, suggesting the need to focus on the role of carotid atherosclerosis in the development of acute stroke. As cerebral infarction is a multifactor disorder resulting from the interaction between environmental and genetic factors, further research into the development and validation of reliable indicators of the highrisk individual is greatly needed.

\section{Disclosure}

The authors report no conflicts of interest in this work.

\section{References}

1. Aho K, Harmsen P, Hatano S, Marquardsen J, Smirnov VE, Strasser T. Cerebrovascular disease in the community: results of a WHO collaborative study. Bull World Health Organ. 1980;58(1):113-130.

2. Mozaffarian D, Benjamin EJ, Go AS, et al. Heart disease and stroke statistics-2016 update: a report from the American Heart Association. Circulation. 2016;133(4):e38-e360.

3. Vickrey BG, Rector TS, Wickstrom SL, et al. Occurrence of secondary ischemic events among persons with atherosclerotic vascular disease. Stroke. 2002;33(4):901-906.

4. Krishnamurthi RV, Moran AE, Feigin VL, et al. Stroke prevalence, mortality and disability-adjusted life years in adults aged 20-64 years in 1990-2013: data from the Global Burden of Disease 2013 Study. Neuroepidemiology. 2015;45(3):190-202.

5. [No authors listed]. National clinical guidelines for stroke: a concise update. Clin Med (Lond). 2002;2(3):231-233.

6. MohanKM, WolfeCD, RuddAG,HeuschmannPU,Kolominsky-Rabas PL, Grieve AP. Risk and cumulative risk of stroke recurrence: a systematic review and meta-analysis. Stroke. 2011;42(5):1489-1494.

7. Markl M, Harloff A. Letter by Markl and Harloff regarding article, "Aortic arch plaques and risk of recurrent stroke and death". Circulation. 2010;121(2):e11; author reply e12.

8. Selwaness M, Bos D, van den Bouwhuijsen Q, et al. Carotid atherosclerotic plaque characteristics on magnetic resonance imaging relate with history of stroke and coronary heart disease. Stroke. 2016;47(6): 1542-1547.

9. Amarenco P, Duyckaerts C, Tzourio C, Henin D, Bousser MG, Hauw JJ. The prevalence of ulcerated plaques in the aortic arch in patients with stroke. N Engl J Med. 1992;326(4):221-225.

10. Amarenco P, Cohen A, Tzourio C, et al. Atherosclerotic disease of the aortic arch and the risk of ischemic stroke. N Engl J Med. 1994;331(22): 1474-1479.

11. Hennerici M, Hülsbömer HB, Hefter H, Lammerts D, Rautenberg W. Natural history of asymptomatic extracranial arterial disease. Results of a long-term prospective study. Brain. 1987;110(Pt 3):777-791.

12. Amarenco P, Lavallee PC, Labreuche J, et al. Prevalence of coronary atherosclerosis in patients with cerebral infarction. Stroke. 2011;42(1): 22-29.

13. Lisabeth LD, Howard G. The current state and future of stroke: introduction. Stroke. 2013;44(6 Suppl 1):S122.

14. Spagnoli LG, Mauriello A, Sangiorgi G, et al. Extracranial thrombotically active carotid plaque as a risk factor for ischemic stroke. JAMA. 2004; 292(15):1845-1852. 
15. Liu XS, Zhao HL, Cao Y, Lu Q, Xu JR. Comparison of carotid atherosclerotic plaque characteristics by high-resolution black-blood MR imaging between patients with first-time and recurrent acute ischemic stroke. AJNR Am J Neuroradiol. 2012;33(7):1257-1261.

16. Wang J, An Z, Li B, et al. Increasing stroke incidence and prevalence of risk factors in a low-income Chinese population. Neurology. 2015; 84(4):374-381.

17. Zhao D, Liu J, Wang W, et al. Epidemiological transition of stroke in China: twenty-one-year observational study from the Sino-MONICABeijing Project. Stroke. 2008;39(6):1668-1674.

18. Chen H, Hong H, Xing S, et al. Intracranial versus extracranial symptomatic carotid atherosclerosis in chinese patients: risk factors, stroke mechanisms, and long-term prognosis. J Stroke Cerebrovasc Dis. 2015;24(11):2632-2639.

19. Liu C. Association of anterior circulation recurrent cerebral infarction and carotid atherosclerotic plaques. Chin J Misdiagn. 2007; 7(24):5746-5747.

20. Zhu X. Clinical study of the association between recurrent cerebral infarction and carotid atherosclerotic. Pract J Clin Med. 2011;15(17): 136-137.

21. Yang M, Chen H. Research on the association between carotid atherosclerotic and recurrent cerebral infarction. Clin Focus. 2007;22(13): 949-950.

22. Zhao D. Association between carotid atherosclerotic and recurrent cerebral infarction. Guide China Med. 2011;09(22):315-316.

23. Yang J, Lin Q. Association between carotid atherosclerotic and recurrent cerebral infarction. J Clin Exp Med. 2008;7(1):25-26.

24. Cao X. Clinical study of the association between carotid atherosclerotic and recurrent cerebral infarction. China Med Herald. 2007; $4(16): 37$.

25. Zhao K, Ding X, Tang Y. Association between carotid atherosclerotic and recurrent cerebral infarction. Mod Med Health. 2011;27(24):3742.

26. Liu L. Association between carotid atherosclerotic plaque and recurrent cerebral infarction. Shandong Med J. 2004;44(28):41-42.

27. Zhang M, Hu Z. Association between carotid artery stenosis and recurrent cerebral infarction. Chin J Coal Ind Med. 2008;11(3):322-323.

28. Zhang M. Comparison of carotid atherosclerotic plaques between recurrent and first-ever cerebral infarction. China J Mod Med. 2007; 17(1):83-85.

29. Wang M. Association between recurrent cerebral infarction and carotid atherosclerosis, plasma Hcy and urinary inhibition-c. Shaanxi Med J. 2010;39(7):859-860.

30. Tan X. Comparison of carotid atherosclerotic plaques between recurrent and first-ever cerebral infarction. China Pract Med. 2009; 4(9):121-122.

31. Wu S. Analysis of risk factors of recurrent cerebral infarction and distribution of vulnerable carotid atherosclerotic plaques [master's thesis]. Shantou University; 2011.

32. Wu J, Gao J. The effect of color ultrasound on carotid atherosclerosis plaque and recurrent cerebral infarction. Anhui Med Pharmaceut J. 2011;15(3):345-347.

33. Song J. Clinical observation of recurrent cerebral infarction. J Dis Monit Control. 2009;3(9):539-541.

34. Zeng G, Shao Y, Zhang C. Relationship between carotid atherosclerotic and recurrent cerebral infarction. J Clin Neurol. 2005;18(1):61-62.

35. Guo H, Guo G, Lin S. Association between recurrent cerebral infarction and carotid atherosclerotic plaque and its risk factors. Heilongjiang Med J. 2012;36(5):326-328.

36. Hua J, Zhou Q. The relationship of homocysteine, carotid artery atherosclerosis and recurrent cerebral infarction. Chin J Pract Nerv Dis. 2012;15(15):3-5.

37. Li J, Zhou W, Peng H. Comparative analysis on related influence factors of primary and recurrent cerebral infarction. Clin J Med Office. 2012; 40(2):320-321.

38. He S, Teng G, Li D. Association between carotid artery atherosclerosis and recurrent cerebral infarction. China Health Care Nutr. 2012; $09: 3162$.
39. Yi X, Yu C, Pan G, Zhang S. The effects of carotid arteriosclerosis on cognitive functions and recent prognosis in patients with cerebral infarction. Chin J clin psychol. 2011;10(1):20-22.

40. Hankey GJ, Jamrozik K, Broadhurst RJ, et al. Five-year survival after first-ever stroke and related prognostic factors in the Perth Community Stroke Study. Stroke. 2000;31(9):2080-2086.

41. De Bruin ML, Dorresteijn LD, van't Veer MB, et al. Increased risk of stroke and transient ischemic attack in 5-year survivors of Hodgkin lymphoma. J Natl Cancer Inst. 2009;101(13):928-937.

42. Mohr JP, Albers GW, Amarenco P, et al. American Heart Association Prevention Conference. IV. Prevention and rehabilitation of stroke. Etiology of stroke. Stroke. 1997;28(7):1501-1506.

43. Howard DPJ, van Lammeren GW, Rothwell PM, et al. Symptomatic carotid atherosclerotic disease: correlations between plaque composition and ipsilateral stroke risk. Stroke. 2015;46(1):182-189.

44. Gloviczki ML, Glockner JF, Lerman LO, et al. Preserved oxygenation despite reduced blood flow in post-stenotic kidneys in human atherosclerotic renal artery stenosis. Hypertension. 2010;55(4): 961-966.

45. Insull W Jr. The pathology of atherosclerosis: plaque development and plaque responses to medical treatment. Am J Med. 2009;122(1 Suppl): S3-S14.

46. Goldschmidt-Clermont PJ, Dong C, Seo DM, Velazquez OC. Atherosclerosis, inflammation, genetics, and stem cells: 2012 update. Curr Atheroscler Rep. 2012;14(3):201-210.

47. Tan CL, Sinha R, Budohoski K, Trivedi RA. MR imaging of vulnerable carotid atherosclerotic plaques. In: Trivedi R, Saba L, Suri JS, editors. 3D Imaging Technologies in Artherosclerosis. New York: Springer; 2015: $171-185$.

48. Libby P. Atherosclerosis: disease biology affecting the coronary vasculature. Am J Cardiol. 2006;98(12A):3Q-9Q.

49. Sadat U, Li ZY, Young VE, et al. Finite element analysis of vulnerable atherosclerotic plaques: a comparison of mechanical stresses within carotid plaques of acute and recently symptomatic patients with carotid artery disease. J Neurol Neurosurg Psych. 2010;81(3):286-289.

50. Zhang AJ, Zhang AY, Zhong C. Carotid atherosclerosis in ischemic cerebrovascular patients. J Clin Med Res. 2009;1(1):40-44.

51. Chambless LE, Folsom AR, Davis V, et al. Risk factors for progression of common carotid atherosclerosis: the Atherosclerosis Risk in Communities Study, 1987-1998. Am J Epidemiol. 2002;155(1):38-47.

52. O’Leary DH, Polak JF, Kronmal RA, Manolio TA, Burke GL, Wolfson SK Jr. Carotid-artery intima and media thickness as a risk factor for myocardial infarction and stroke in older adults. Cardiovascular Health Study Collaborative Research Group. N Engl J Med. 1999; 340(1):14-22.

53. Persson J, Formgren J, Israelsson B, Berglund G. Ultrasound-determined intima-media thickness and atherosclerosis. Direct and indirect validation. Arterioscler Thromb. 1994;14(2):261-264.

54. Wong M, Edelstein J, Wollman J, Bond MG. Ultrasonic-pathological comparison of the human arterial wall. Verification of intima-media thickness. Arterioscler Thromb. 1993;13(4):482-486.

55. Touboul PJ, Elbaz A, Koller C, et al. Common carotid artery intimamedia thickness and brain infarction: the Etude du Profil Génétique de l'Infarctus Cérébral (GENIC) case-control study. The GENIC Investigators. Circulation. 2000;102(3):313-318.

56. Nagai Y, Kitagawa K, Sakaguchi M, et al. Significance of earlier carotid atherosclerosis for stroke subtypes. Stroke. 2001;32(8):1780-1785.

57. Ziegelbauer K, Schaefer C, Steinmetz H, Sitzer M, Lorenz MW. Clinical usefulness of carotid ultrasound to improve stroke risk assessment: ten-year results from the Carotid Atherosclerosis Progression Study (CAPS). Eur J Prev Cardiol. 2013;20(5):837-843.

58. Lukasik M, Rozalski M, Luzak B, et al. Enhanced platelet-derived microparticle formation is associated with carotid atherosclerosis in convalescent stroke patients. Platelets. 2013;24(1):63-70.

59. Markus HS, Makela KM, Bevan S, et al. Evidence HDAC9 genetic variant associated with ischemic stroke increases risk via promoting carotid atherosclerosis. Stroke. 2013;44(5):1220-1225. 
60. Liu ME, Liao YC, Lin RT, et al. A functional polymorphism of PON1 interferes with microRNA binding to increase the risk of ischemic stroke and carotid atherosclerosis. Atherosclerosis. 2013;228(1):161-167.

61. Arntzen KA, Schirmer H, Johnsen SH, Wilsgaard T, Mathiesen EB. Carotid atherosclerosis predicts lower cognitive test results: a 7-year follow-up study of 4,371 stroke-free subjects - the Tromsø study. Cerebrovasc Dis. 2012;33(2):159-165.
62. Romero JR, Beiser A, Seshadri S, et al. Carotid artery atherosclerosis, MRI indices of brain ischemia, aging, and cognitive impairment: the Framingham study. Stroke. 2009;40(5):1590-1596.

\section{Publish your work in this journal}

Neuropsychiatric Disease and Treatment is an international, peerreviewed journal of clinical therapeutics and pharmacology focusing on concise rapid reporting of clinical or pre-clinical studies on a range of neuropsychiatric and neurological disorders. This journal is indexed on PubMed Central, the 'PsycINFO' database and CAS, and is the official journal of The International Neuropsychiatric Association (INA). The manuscript management system is completely online and includes a very quick and fair peer-review system, which is all easy to use. Visit http://www.dovepress.com/testimonials.php to read real quotes from published authors.

Submit your manuscript here: http://www.dovepress.com/neuropsychiatric-disease-and-treatment-journal 